\title{
Psychosomatic Molecular Mechanism of Metabolic Syndrome and Type 2 Diabetes. Part 3. Is Type 2 Diabetes a Decompensated Form of Metabolic Syndrome?
}

\author{
András Sikter MD* \\ Internal Medicine, Municipal Clinic of Szentendre, Szentendre, Hungary, Europe \\ *Corresponding Author: András Sikter MD, Internal Medicine, Municipal Clinic of \\ Szentendre, Szentendre, Hungary, Europe. E-mail: andrassikter3@gmail.com
}

DOI: $10.31080 /$ ASMS.2020.04.0553
Received: January 17, 2020

Published: February 07, 2020

(C) All rights are reserved by András Sikter.

\begin{abstract}
Insulin is one of the most important of anabolic hormones. Type 2 Diabetes (T2D) is characterized by persistent, stealth catabolism, as well as impaired glucose and phosphate uptake. The phosphate transporting capability of insulin has been known for a long time, although it has been studied mainly since the $21^{\text {st }}$ century. According to the logic of the Momentary Intracellular Ion-Pattern Signaling, insulin should increase of $\mathrm{HPO}_{4}^{2-}$ in the cytosol - and does it, indeed. (It is not clear that this is a one- or multistep membrane-transport phenomenon.) Insulin does not only stimulate the transport of phosphate (Pi) into the cytosol but alkalinizes it, as well. Cytoplasmic alkalinization is a vital process by insulin, which mechanism has not been studied in detail. Insulin is one of the most important players in the regulation of intracellular $\mathrm{pH}$, which, together with phosphate transport, is crucial for maintaining its anti-catabolic effect. Acidotic cytoplasm of the pancreas beta-cells increases while alkalotic decreases insulin secretion, which may be a feedback mechanism.
\end{abstract}

All insulin-mediated vital anabolic processes use energy derived from ATP. The impaired mitochondrial ATP production capability is a consequence of insulin resistance, which develops during the early stage of Metabolic Syndrome, and can persist for decades without a manifest of T2D. Decreased ATP productivity is compensated by increased lipid availability. T2D develops when high triglyceride availability cannot compensate for insulin resistance and reduced ATP production. The guiding principle is that mitochondria have to provide near-normal ATP content at all costs in the cytoplasm of the cells; otherwise, the cells will be severely ill or killed. Catabolism rises to increase ATP production. The liberated amino acids allow gluconeogenesis to proceed and raise serum glucose levels. Pathophysiologically, both hyperglycemia and increased food uptake have compensatory roles in ATP production, though many vicious circles are generated.

Keywords: Chronic Low-Grade Hypercapnia; Cytosolic Alkalinization; Insulin Mediated Pi-Transport; Insulin Resistance; Intracellular Acidosis; Type 2 Diabetes

\section{Abbreviations}

ATP: Adenosine Triphosphate; BCM: Body Cell Mass; BMI: Body Mass Index; CBIs: Cytoplasm Builder Ions; COPD: Chronic Obstructive Pulmonary Disease; DAG: Diacetyl Glycerol; FFA: Free Fatty Acid; G-6-P: Glucose-6-Phosphate; GLUT4: Glucose Transporter Type 4; IGF-I: Insulin -Like Growth Factor-1; IL-6: Interleukin-6; IMCL: Intramyocellular Lipid; IR: Insulin Resistance; LBM: Lean
Body Mass; MetS: Metabolic Syndrome; MRS: Magnetic Resonance Spectroscopy; NHE: Natrium-Hydrogen Exchanger $=\mathrm{Na}^{+} / \mathrm{H}^{+}-\mathrm{Ex}-$ changer; OHS: Obesity Hypoventilation Syndrome; OSA: Obstructive Sleep Apnea; OSAS: Obstructive Sleep Apnea Syndrome; $\mathrm{pCO}_{2}$ : Partial Pressure of Carbon Dioxide; pHi: Intracellular pH; Pi: Inorganic Phosphates; ROS: Reactive Oxygen Species; RQ: Respiratory Quotient; T2D: Type 2 Diabetes; TG: Triglycerides; TNF: Tumor Necrosis Factor 


\section{Introduction}

Insulin is one of the most important of anabolic hormones. Type 2 Diabetes is characterized by persistent, stealth catabolism, as well as impaired glucose and phosphate uptake. The primary targets of insulin are on the cells of the liver, skeletal muscle, and fat tissue. Besides, most cells of the organism also have insulin receptors, where it usually has pleiotropic effects [1]. All insulin-mediated vital processes use energy derived from ATP. The primary metabolic "goal” of insulin is to provide the conditions of the ATP production on a high level, as it is a prerequisite for both its anabolic and pleiotropic actions. The vital anabolic processes supported by insulin are the following: protein synthesis/anabolism, phosphorylation, growth/mitogenesis, reproduction, lifespan, cognition [1]. Mitochondrial ATP production is already impaired at an early stage of insulin resistance (IR) [2,3] These persons can be asymptomatic for more decades despite Metabolic Syndrome develops. Hypertriglyceridemia and cytoplasmic accumulation of lipids start when IR develops - lipids can play a compensatory role in metabolism for a long time. It was presumed [3] that the intramyocellular lipid (IMCL) evolves due to a probable genetic defect of mitochondrial ATP production.

There are several causes and putative pathomechanisms for the development of T2D. While previously, the lipid theory used to lead the list, high levels of serum lipids are increasingly considered to be a compensatory phenomenon and, therefore, is a good predictor of the IR. According to the author of this book, each of the putative pathogenetic factors also can trigger acidosis. T2D is likely to develop from MetS when increased lipid availability is no longer able to provide sufficient ATP production on a sustained basis, and the cytosolic ATP level begins to decline. The lack of CBIs $\left(\mathrm{Mg}^{2+}, \mathrm{Zn}^{2+}, \mathrm{Pi}\right.$, and $\left.\mathrm{K}^{+}\right)$also plays a significant role in the development of T2D (see Part 4). Previously less discussed, however, it has been shown that $\mathrm{T} 2 \mathrm{D}$, as a slow catabolic process, is associated with decreased LBM and BCM (See Part 1), so-called Sarcopenic Obesity often occurs.

The author suggests that there may be only predisposing genetic factors in the background rather than significant genetic defects, given that IR is a widespread phenomenon. Pi is a cytosolic signaling molecule, which can control the metabolic rate through the regulation of oxidative phosphorylation [4]. The insulin acts on a specific cell membrane receptor, which belongs to the super- family of tyrosine kinases, activates a complex intracellular signaling network and cascades. Some researchers have suggested that impaired ATP production of mitochondria (and IR) can develop due to intracellular acidosis and is a consequence of inadequate phosphate transport. Psychosomatic pathomechanisms can also cause it (see Part 2). We cannot alter either the assumed genetic errors causing IR or chronic stress, which already has taken place. However, the metabolic alterations based on inorganic chemical modifications caused by chronic hypercapnic acidosis can be partly or wholly reversed, and they can be corrected by physicians, at least that's what the author of this E-Book thinks.

About insulin resistance, Type 2 Diabetes and their metabolic consequences

Phosphate transport defect is an early sign of insulin resistance

Insulin physiologically facilitates not only the membrane transport of glucose but also promotes the phosphorylation of glucose to G--6-P [5]. Insulin (similarly to IGF-I) helps the transport of inorganic phosphate into cells by $\mathrm{Na}^{+} / \mathrm{Pi}$ cotransport mechanism [6]. One of the most significant consequences of IR is the decrease of cytoplasmic Pi concentration in muscles, diminished glucose phosphorylation [5], and other phosphorylations [7]. Petersen also suggests that the two defects root in the same problem [2]. High plasma levels of fatty acids are a good predictor of IR $[7,8]$.

Many think that two articles of Petersen's team [2,3] are the cornerstones of IR. They showed that insulin-resistant young, lean, apparently healthy offspring, had already impaired mitochondrial activity [2]. According to prospective studies, IR can be decades before the onset of diabetes manifestation [9]. The insulin-resistance and insulin-sensitive subjects showed similar characteristics in age, weight, BMI, activities, glycosylated hemoglobin [2]. Insulin levels during oral glucose tolerance tests were consequently higher than controls. Their data showed (during proton magnetic resonance spectroscopy) that the insulin-stimulated rate of glucose uptake by muscle was $60 \%$ lower than in the controls ( $\left.\mathrm{P}^{*}<0.001\right)$. It was accompanied by an increase of $80 \%$ in IMCL. (P = 0.005). The decrease in mitochondrial phosphorylation was 30\% ( $\mathrm{P}=0.01)$. (Note: This condition can be consistent with MetS.) Their conclusion: There is a defect in mitochondrial oxidative phosphorylation in the skeletal muscle of the insulin-resistance offspring patients with T2M, which may be inherited. IR manifests with increased 
IMCL and decreased insulin-stimulated glucose uptake. In the second paper of Petersen's team [3], they pointed out that, in addition to the reduction of insulin-stimulated mitochondrial ATP synthesis, the insulin-stimulated phosphate transport decrease also clearly detectable. There was a robust correlation between an insulin-stimulated intramyocellular Pi increase and the rates of ATP synthesis. The authors declare: "a common signaling defect may be responsible" for both intramyocellular Pi and glucose transport defects. Also, it should be investigated which one is the primary of the three (increased IMCL, decrease in ATP synthesis, and Pi transport), and which are consequences? According to the logic of the cytoplasmic model (see Part 1.), the decreased Pi transport should be the primary cause of the reduced ATP synthesis. Pi transport is hindered due to hypercapnia induced intracellular acidosis. The increase in IMCL is probably a compensatory phenomenon [10].

Today, there is a growing acceptance that damaged ATP formation, which results from decreased Pi transport, can be the direct cause of IR. The so-called "subsarcolemmal mitochondria" obtained from obese patients by biopsy, the mitochondrial electron transport chain activity (ATP generation) was significantly lower than in lean, healthy subjects [11]. It would mean that IR is present in a part of the mitochondria of a significant proportion of obese patients. Naturally, not all obese patients will have diabetes. There is an age-related reduction in mitochondrial ATP production capacity in skeletal muscles. That is why IR is common in the elderly, which is often not manifested in the classic clinical picture of T2D. Following the classical MRS examinations of the Petersen's group, their results and theory were repeatedly confirmed. Investigating the acute effect of insulin on healthy human skeletal muscle, it became unequivocal that ATP content started to increase after 50 minutes administering of insulin and lasted for 150 minutes [12]. Pi uptake into the muscle was $14 \%$ while glucose uptake $8 \%$. The insulin-mediated increase in ATP concentration was entirely due to the rise in Pi transport. In summary, in the case of IR, the insulin-stimulated ATP synthesis reduced due to the decreased insulin-stimulated Pi transport.

\section{Role of lipids in the insulin resistance}

Decreased oxidative capacity of skeletal muscle can be significant contributors to the development of IR and T2D because it is one of the main ATP generating organs, considering its volume too. There is evidence for increased lipid content in skeletal muscle (IMCL) in IR: histology, electron microscopy of biopsies, and mag- netic resonance spectroscopies are also available. An impaired ATP synthesis and increased IMCL were also present in lean offspring of type 2 diabetes as an inborn defect of oxidative phosphorylation [2]. Under experimental insulin-stimulated conditions, increased serum lipid concentrations moderated the insulin-stimulated ATP synthase flux and impaired glucose-transport/phosphorylation. Elevation of plasma free fatty acid (FFA) concentrations reduces glucose transport, phosphorylation, glycogen synthesis, and glucose oxidation. On the other hand, acute increases in plasma FFAs cause increases in intramyocellular triglyceride (IMCL-TG) content in healthy volunteers. However, novel data suggested that the lipids induced IR is less straightforward than has been previously suggested. The lipid overflow hypothesis is overshadowed because researchers have pointed towards multiple factors that defense against the development of IR. The lipid droplet coat proteins protect the cytoplasm from IMCL lipotoxic effects. Although the disbalance in skeletal muscle lipid influx can be toxic. The observation of the mechanisms involved in lipid-induced IR is not yet closed. E. g., DAG, ceramides can induce IR in experimental animals. Such mechanisms could not be proven clearly in human studies. On the other hand, there has been found that there are several routes to lipid-induced IR [13]. Today, the majority accepts that lipotoxicity becomes manifest, at least concerning pancreatic beta-cells, if glucotoxicity is also present. Hyperglycemia itself is toxic for beta-cell function [14].

T2D is a heterogeneous disorder. The disordered lipid metabolism is neither primarily nor responsible solely for the development of IR [2,3]. Metabolic Syndrome (MetS) and Type 2 Diabetes (T2D) are clinical conditions involving impaired uptake and utilization of glucose and altered lipid metabolism [8]. It seems that at the beginning of IR, in the MetS stage, mitochondrial lipid oxidation can compensate the inhibited glucose uptake. Serum lipid levels increase due to impaired mitochondrial ATP generation; this is a compensatory phenomenon [10], that is why the serum lipid level elevation is a good predictor of IR $[7,8]$. Already at the stage of MetS, the elevated serum lipid levels are followed by the increase of intracellular lipid contents (e.g., IMCL); these can increase oxidative phosphorylation and maintain an acceptable level of ATP formation by increasing Acetyl Coenzyme A levels. The dominance of lipid oxidation is also prooved by a high respiratory quotient (RQ) [8]. Subsequently, however, due to vicious circles, this lipid compensation becomes inadequate, and T2D develops. One of the reasons for this can be

Citation: András Sikter. "Psychosomatic Molecular Mechanisms of Metabolic Syndrome and Type 2 Diabetes. Part 3. Is Type 2 Diabetes a Decompensated Form of Metabolic Syndrome?". Acta Scientific Medical Sciences 4.3 (2020): 10-23.

DOI: $10.31080 /$ ASMS.2020.04.0553

Published: February 07, 2020 
the incomplete fatty acid oxidation and the mitochondrial overload itself [15]. The onset of T2D initiates a series of vicious circles (see below) - one of the consequences is that the lipid oxidation further deteriorates [8].

The increased serum lipid levels and the resulting increased IMCL are not highly toxic for years, decades, and they provide adaptive compensations for IR and decreased glucose oxidation. However, it should be some reason that, over time, lipid beta-oxidation also becomes insufficient. By the time developing T2D, the pancreatic beta-cells are already severely damaged [16]. It should explain what the pathomechanism might be, Koves' team experiments can help us in these issues [15]. They found that lipid beta-oxidation is enhanced in IR, at least in the skeletal muscle cells. Their extensive researches support the theory of "incomplete" degradation of lipids during excessive beta-oxidation, which can eventually impair mitochondria, ultimately leading to a decrease in energy deficiency and decreased membrane potential. In short: after prolonged existence, increased lipid oxidation becomes toxic, especially in the presence of glucose. That is, the poisonous effect of lipid overload will occur sooner or later [15].

Role of beta-cell dysfunction in the pathophysiology of Type 2 Diabetes

Beta-cells of the pancreas are capable at first secrete sufficient insulin to compensate for IR, then relative insulin deficiency develops. When hyperglycemia exists, beta-cell dysfunction is already clearly present in persons with Type 2 Diabetes [16]. Many different studies confirmed that beta-cells dysfunction occurs well before the onset of hyperglycemia. Most researchers assigned to persistent lipotoxicity as causative for the relatively reduced insulin production, others claim that only the glucotoxicity makes high lipid levels also toxic [14]. The discussion has not yet settled what causes pancreatic beta islet cells' early damage. Kahn [16] also regards the pathogenesis of T2D as a vicious circle. The combination of lipid-induced IR with a weakening insulin response and increased lipolysis. Namely, the account has to be taken the weakening anabolic and increasing catabolic effects of T2D. At the same time, there is a failure of the hypothalamus to induce postprandial thermogenesis [17]. The weakening insulin action increases body mass (BMI) while decreases cytoplasmic weight (BCM.). Reduced rate of energy expenditure is a risk factor for body-weight gain [18].
Since the role of lipotoxicity (without hyperglycemia) has been questioned in the pathogenesis of T2D, there is no accepted pathophysiology for early beta-cell dysfunction [13]. The author of this book accepts Koves' opinion [15] that persistent elevations in serum lipid levels and IMCL have some toxic effects on mitochondria. Frequent polyphagia, because of starvation due to ATP deficiency, both lipid and glucose levels often elevate transiently, which potentiate each other toxic effects. On the other hand, very high levels of $\mathrm{pCO}_{2}$ (in the case of the OSA, with or without hypoxia) also play significant roles.

Cytosolic acidification is a potent candidate for insulin resistance

It was found that GLUT4 glucose trafficking is the rate-controlling step at the insulin-stimulated glycogen synthesis in the skeletal muscle [5]. Although it only functions well under alkaline cytosolic pH conditions.

Previously, (in the early seventies), it was thought that intracellular $\mathrm{pH}(\mathrm{pHi})$ is constant, which was refuted [19]. Interest in $\mathrm{pHi}$ is far ahead of the possibilities. The influence of intracellular $\mathrm{pH}$ on insulin secretion has been known since 70ies-80ies, but little attention has been paid to these mechanisms [20]. Although, there was a debate about whether glucose changes the cytosolic $\mathrm{pH}$ or not when enters to beta-cells? If yes, in which direction? On the other hand, does the NHE $\left(\mathrm{Na}^{+} / \mathrm{H}^{+}\right)$exchanger mechanism play a role in the most commonly detected alkalinization [21]? There is strong evidence that intracellular $\mathrm{pH}$ influences the so-called Nutrient-Stimulated Insulin Secretion [20]. It was identified that glucose activates the vacuolar V-ATPase (in yeast and mammals) and alkalinizes the cytosol [22]. A Chinese research group (Nankai University) looks like, solved the $\mathrm{pH}$-dependent, voltage-gated channel regulation of insulin secretion, reported in an unpublished paper [23].

Voltage-gated proton channels are membrane proteins; they allow only protons to cross membranes. They located in all cells from unicellular to mammals. They allow protons to move if the electrochemical gradient is outwards. These membrane proteins are "rectifiers." They are sensing the $\mathrm{pH}$, are also changing the intracellular $\mathrm{pH}$ due to the flow of the protons. Voltage-gated channels are sometimes closed depending on the voltage- and $\mathrm{pH}$-gradient on the two sides of the membrane [24]. It was demonstrated on pancreatic beta-cells that the secretory granules of vacuoles need aci-

Citation: András Sikter. "Psychosomatic Molecular Mechanisms of Metabolic Syndrome and Type 2 Diabetes. Part 3. Is Type 2 Diabetes a Decompensated Form of Metabolic Syndrome?". Acta Scientific Medical Sciences 4.3 (2020): 10-23.

DOI: $10.31080 /$ ASMS.2020.04.0553

Published: February 07, 2020 
dification driven by V-type $\mathrm{H}^{+}$-ATPase to secrete insulin [25]. It was identified that glucose activates the vacuolar V-ATPase (in yeast and mammals) and alkalinizes the cytosol, vacuolar and cytosolic $\mathrm{pH}$ alterations were second messengers for glucose in beta-cells [22]. The alkalinization of the cytoplasm of beta-cells inhibited insulin secretion [26]. Although, cytosolic acidification stimulated the insulin release from beta-cells [27]. Manipulations of $\mathrm{pH}$ in the pancreas beta-cells are associated with changes in membrane potential, ionic fluxes, and insulin release [23]. Relatively small changes in Hv1 activity have significant effects in insulin secretion, which that is the voltage-gated system is an excellent regulator of metabolism [23]. Pang., et al. [23] seem to have clarified an essential regulatory mechanism for insulin secretion. It can take time for the previously discovered mechanisms $\left(\mathrm{K}_{\mathrm{ATP}}\right.$ channel, the role of $\mathrm{Ca}^{2+}$ ) to retake to their places.

What does mean the newly discovered mechanism of insulin formation, the voltage-gated insulin secretion? The author of this book thinks that insulin regulates not only the level of blood glucose, but it is also one of the intracellular pH regulators. Namely, it tries to prevent the acidification of the cytosol. The feedback mechanism also works here: insulin alkalinizes the cytoplasm of target cells. If the medium is sufficiently alkaline, the cytoplasm of the beta-cells will also be more alkaline (at least in its tendentiously). But if the $\mathrm{pH}$ of the cytosol is more acidic, more insulin will be produced to combat acidosis, as is the case in the Metabolic Syndrome; and this works indeed for many years. Although this pHi regulation cannot be perfect, in part because insulin receptors are represented in very different proportions on different cell types. On the other hand, respiratory acidosis cannot be compensated by ion-exchanger so, such that Intracellular Ion Pattern set to the original level (see Part 2.).

It cannot be overemphasized - and it is no overstated - that there is a strong relationship between acidosis and insulin metabolism or insulin receptor signaling [28]. Surprisingly few publications deal with the role of acidosis in IR, much of them on diabetic ketoacidosis, and are old papers. However, it has been established [29] that metabolic acidosis was more connected to the occurrence of IR than metabolic ketosis. They suggested that IR in metabolic acidosis resulted from the combined defects in binding affinity and the post-binding process on the insulin receptor. The metabolic acidosis but not ketosis caused IR in the rat [30]. Nephrologists have long been recognized that metabolic acidosis due to chronic renal failure causes IR, which was reduced by the restoration of serum $\mathrm{pH}$ [31]. The insulin production increased with the disappearance of extracellular acidosis, which was explained with an increase in vitamin D3 levels. (It may be a contradiction.).

Marunaka [32] found a slight decrease in interstitial fluid $\mathrm{pH}$ of the body. According to his theory, the buffering capacity of interstitial space is lesser than blood plasma. The overproduction of acid metabolites would lower the $\mathrm{pH}$ of interstitial fluids. The lowered pH (acidosis) could cause IR. With a modern Western-type diet, we consume a lot of titratable acids. Research results suggest that the acid load taken with meals can be an additive mechanism for the development of IR [33].

There is no consensus as to what is the primary cause of IR. The three leading candidates include 1) intracellular fatty acid accumulation, chiefly in the cytoplasm of the liver, skeletal muscle, and heart, 2) dysfunction/failure or decreased number of the mitochondria, 3) inflammatory mechanisms, primarily provoked by the cytokines of adipose tissue (as TNF-alpha and IL-6). Other factors also emerged, for example, the ROS. Recently, the role of acidosis has even started to spread (see above). The author of this book believes that it does not take too much effort to bring the causative factors to a common denominator, namely, to prove therole of intracellular acidosis in all proposed mechanisms, which consequences from the cytoplasm model of the author, too (see Part 1.).

To point 1): Hamilton had accurately described that all FFAs easily cross the membrane of cells (according to his flip-flop concepts), they acidify the cytosol for a while, then will be neutralized relatively fastly. It is a consequence of the acidic manifestation of the non-esterified fatty acids. The neutralization by cytoplasmic proteins follows it. It was shown that adipocytes are much less able to absorb and internalize certain long-chain fatty acids if they were manipulatively acidified previously. On the other hand, the $\mathrm{pH}$ could be restored much more slowly [34]. That is, fatty acids can be toxic because they can acidify cytosol.

Many say that the primary cause of lipid accumulation in the cytoplasm is the inadequate fat burning [10]. According to this theory, both glucose and lipids exist in higher serum concentrations because their burning is hindered, and the steady-state can only be 
created at higher levels - or neither then. The cells are starving in T2D, so the individuum is also starving. The more calories he/she takes in, the worse the situation will be. (A newer vicious circle.) NB. Biochemical processes can only utilize biological energy (ATP).

The electrochemical gradient of $\mathrm{H}^{+}$points to the direction of the cytoplasm (see Part 2. [30]), and therefore the extrusion of the acids from the cytosol requires direct or indirect ATP energy. The fatty acids will undoubtedly cause a significant acidic load. In the case of ATP deficiency, the acid removal will be damaged, so long term cytosolic acidosis will develop.

To point 2): An early paper on IR reflects a modern view, when writes: It is valid of the "insulin resistance of skeletal muscle in type 2 diabetes and obesity dysregulation of both carbohydrates and lipid fuels" [35]. Since then, several mitochondrial morphological and functional disorders have been shown to play a role in the development of IR. Many of these appear to be hereditary. Either way, something had to change in a hundred years because, in the earlier centuries, T2D might be a less frequent disease, though the genome could not change significantly. (Some think that mitochondrial damage may be a consequence of IR [36]. One of the consequences of the relative or absolute ATP-energy insufficiency is the Sick Cell Syndrome (see Part 1), which includes, among other things, intracellular acidosis and phosphate deficiency. Although in the case of T2D, the shortage of Pi transport is not causative, the cause itself (see Part 4.).

To point 3): The proinflammatory cytokines as tumor necrosis factor (TNF) and interleukins 1, 6, 8 (IL-1, IL-6, IL-8) are initiators of the acute phase reaction. They heighten catabolism, cause negative nitrogen, potassium, magnesium, and phosphate balance, as well as acidosis and IR [37]. There is an apparent antagonistic effect between anabolic hormones and proinflammatory cytokines. Insulin-like growth factor-1 (IGF-I) could reduce TNF- $\alpha$ or IL-1 $\beta$-induced proteoglycan degradation [38]. Anabolic action of insulin is similar to IGF-I. The relationship is evident. It also means that inflammation also causes "Sick Cell Syndrome" and intracellular acidosis (See Part 1).

The above speculative reasoning was possible because, currently, reliable cytosolic $\mathrm{pH}$ measurements are not available in clinical practice. The author of this book agrees with the following.
"However, next-generation techniques allow for the real-time measurement of live-cell metabolism, intracellular $\mathrm{pH}$, and proton pumping, both through imaging instrumentations and biochemical assays, thereby opening up the possibility for a better comprehension of the phenomenon for the future" [28].

Chronic respiratory acidosis, together with metabolic acidosis, can generate Metabolic Syndrome and Type 2 Diabetes

According to the author of this book, T2D is most often triggered by low-grade chronic hypoventilation/hypercapnia. OSA, COPD, and OHS play a crucial role in the development of IR because of taking part in the pathophysiology of chronic (but fluctuating) hypercapnia (see Part 2). Hypercapnia generates intracellular acidosis, and that low-grade acidosis is able to cause catabolism (See Part 1., and Part 2). Surprisingly, the catabolic effect of hypercapnia was discovered only in the $21^{\text {st }}$ century [39]. According to the hypothesis of this book's author, slight chronic hypercapnia would be not only the etiopathogenetic factor that of T2D but also that of MetS. Chronic stress plays a crucial role in the pathophysiology of all three hypoventilation syndromes (OSA, COPD, and OHS). Still, many others also participate: for example, age, eating habits, traditions, psychic mechanisms (rewarding), lack of movement. The individual determinants, partly genetic that are also decisive how the course of the disease at the person.

The author had shown (see Part 2.) how this type of chronic stress can transform the intracellular $\mathrm{pH}$ and ion-relations through breathing changes and stress hormones. (Stress -- breathing alteration -- $\mathrm{CO}_{2}$ level alteration -- pH-alteration -- electrolyte disturbances -- metabolic alterations -- metabolic remodeling.) The neuro-humoral system tries to recover intracellular $\mathrm{pH}$; the riffle effects and vicious circles develop. In the case of this type of chronic stress, chronic hypercapnia persists, compensatory mechanisms can stabilize metabolism. The deterioration can further continue by the dysregulation and remodeling of the metabolism. Restoring the original $\mathrm{pH}$ and ionic conditions together everywhere in the body is a mathematical inability. Assuming original states were optimal, the deterioration of the body will increase, the levels of entropy would equalize sooner than without the existing alterations of $\mathrm{pCO}_{2}$; life will shorten. These conditions, under certain circumstances, are manifested in the form of a clinical picture of T2D. 
In addition to $\mathrm{pH}$ that expresses actual acidity, titratable acidity should also be mentioned. This metabolic component neutralizes the nanomolar $\mathrm{H}^{+}$magnitude alterations of blood, with molar concentrations of buffers. Modifications of the actual acidity are compensated by the kidneys and are buffered by the tissues. (The "complication" term better express the essence than the "compensation" since the metabolic component can recover similarly slowly as it was created. This mechanism helps in maintaining pathological states.) About $1 \mathrm{mEq}$ of an organic acid is produced from every gram of protein consumed. The $\mathrm{mEq} / \mathrm{L}$ unit is a million times bigger than the $\mathrm{nEq} / \mathrm{L}$ (concentration of the dissociated form of $\mathrm{H}^{+}$). It means that the entire amount of the produced acids should be emptied via kidneys or bound by tissue buffers. Otherwise, it would be developed intolerable acidosis. Clinicians recently realized that the largest buffering organ, i.e., the bone system itself also changes and suffers from the acid-binding, and osteopenia can develop as a result [40]. The hydrogen ion can be formed de novo from almost "out of the nothing" (for example, from the metabolic end-products or intermediate products of organic matter) as well as it also can similarly disappear. As opposed to the metal ions which can enter the body (incorporation), or they can leave it by excretion. Hypoxia, ischemia, necrosis - all these processes are accompanied by intense acid formation.

It was not taken into account previously that the buffer itself, in this case, bone tissue, also changes during buffering; binding of fixed acids certainly contributes to osteoporosis. Over the decades, the idea of Wachman and Berstein [40] is increasingly supported by studies [33]. This loss of the bone mass is mostly reversible if we ingest fixed alkaline salts and $\mathrm{Ca}^{2+}$ instead of fixed acids. With the alterations of titrable acidity, the actual acidity also changes, only by many magnitudes lesser. Relman., et al. developed a method that can well follow the acid-base balance. "Where did the endogenous acids normally come from?" [41]. There are many arguments in favor of the theory raised 50 years ago [38], but there are also objections [33]. Sufficient data is available to prove that inadequate nutrition brings so many acids into the body that can impair insulin sensitivity and glucose homeostasis. However, there are no functional studies to prove that these harmful effects can be eliminated. It was stated that the acidogenic diet can cause metabolic acidosis, IR, and metabolic syndrome [33].

Several circumstances, literature studies, suggest that age-re- lated metabolic acidosis, which is partly irreversible, contributes to the formation and maintenance of MetS and T2D (see Part 2).

\section{Dynamics of the metabolism}

Intracellular $\mathrm{pH}$ is one of the essential signaling systems because it affects all enzymes, more or less. A relatively small raise in $\mathrm{pH}$ results in an up to $20 \%$ increase in metabolism (see Part 1. [9]) as it strengthens the activity of a lot of enzymes. By contrast, a minor decrease in intracellular $\mathrm{pH}$ reduces metabolism; thereby, it has a stabilizing effect for a short time. It is preferred in emergency physicians, especially in the form of moderate hypercapnia: "permissive hypercapnia" - (see Part 2. [45]). Indeed, this often prevents, postpones the threat of life, and gives time for action. By contrast, chronic hypercapnia is not at all desirable for the long run even in a moderate form, because the anabolism is more reduced than catabolism; slow degradation of cytoplasm is inevitable. When it comes to intracellular acidosis, it is also important to note that the cell space is not continuous but also fragmented. Cells are not a single space but have many vacuoles, and other cell organelles, which are surrounded by membranes, each of them has its regulation. So it is only guessing to speak about homogenous intracellular acidosis or alkalosis evenly the body. We can imagine it rather mosaic-like, which could explain the validity and pathophysiology of the Place of Less Resistant Theory (see Part 1.). There is no evidence of either of them because measuring intracellular $\mathrm{pH}$ is not solved.

So when we talk about intracellular (or cytosolic) acidosis, we can only say that in general. The author of this book cannot do better to do than is following the cytoplasmic model that he had constructed from the review of countless literary articles through decades ago. The equilibrium of anabolism versus catabolism is sometimes necessary for the stability of life. On the other hand, the cytoplasmatic (or cell) growth is one of the vital life processes [1] supported by the action of insulin. There is a clear correlation between cytosolic $\mathrm{pH}$ and cytoplasmic increase [42]. The increase-decrease dynamics of the cell cytoplasm can best be illustrated by the cytoplasmic triad (CBIs-ATP-Proteins) helix, where the height of the helix is proportional to the amount of each component. It correlates with resting membrane potential (or "potassium potential") and ATP content of the cell in a steady-state. (See Part 1. Figure 1 and 2). Even if only one of the CBIs $\left(\mathrm{K}^{+}, \mathrm{Mg}^{2+}, \mathrm{HPO}_{4}{ }^{2-}, \mathrm{Zn}^{2+}\right)$ decreases or one of "extracellular ions" $\left(\mathrm{Na}^{+}, \mathrm{Ca}^{2+}, \mathrm{Cl}^{-}\right.$or $\left.\mathrm{H}^{+}\right)$amount increases in the cytoplasm, the equalization starts to begin: all CBIs will

Citation: András Sikter. "Psychosomatic Molecular Mechanisms of Metabolic Syndrome and Type 2 Diabetes. Part 3. Is Type 2 Diabetes a Decompensated Form of Metabolic Syndrome?". Acta Scientific Medical Sciences 4.3 (2020): 10-23.

DOI: 10.31080 /ASMS.2020.04.0553

Published: February 07, 2020 
decrease until proportionality develops. The measure of proteins and ATP decreases proportionately as well. The cytosolic acidosis is one of the commonest inducers of catabolism (see Part 1. [33]). While the catabolism is in progress, the CBIs enter the extracellular space; when the catabolism stops, metabolism can rebalance again. By then, the CBIs had excreted out through the kidneys; normal-low CBIs occur initially. Then, during recovery, which is an anabolic process, low levels of CBIs will develop. In other words, CBIs are similar to the icebergs: their larger pieces are inside the cytoplasm, they insert their peaks into the extracellular space. But contrary icebergs they emerge out sometimes better (in catabolism) and sometimes less (in anabolism). (Many other factors also influence serum electrolyte levels.).

In summary, the meaning of momentary serum level of CBIs needs to evaluate actively. It follows from the above: for example, low Se-Mg levels are almost certainly indicative of $\mathrm{Mg}^{2+}$ deficiency, but normal or high Se-Mg levels are not an excess, it may mean catabolism, renal dysfunction, or possibly toxicity too. In the case of T2D, the deficiency of all the CBIs always develop (see Part 4.). Although supplementing only one of them (e.g., $\mathrm{Mg}^{2+}$ ) is rarely successful. Only if it is the limiting factor, as in Golden's cases, the $\mathrm{Zn}^{2+}$ (see Part 1. [23]). That is, for successful supplementation, many conditions must be present together. On the other hand, hardly changing, normal Se-Mg (or other CBIs) levels usually mean steady-state stability, while continually evolving and low Se-Mg (or other CBIs) levels indicate the instability of the metabolism. In this case, they show an imperfect balance of T2D, which can be a good sign because it means there's still room for improvement of metabolism (either with electrolyte replacement or drug interventions).

The process of Metabolic Syndrome decompensation consists of countless vicious circles

In T2D, and also in other diseases, many vicious circles can be identified. In the pathogenesis at the progression of diseases, vicious circles are not exceptions but a law (see Part 1.). Searching on the PubMed database, the author of this book found 160 publications with search terms "diabetes" AND "vicious circle" together, indicating some dozens identified interrelationships. Factors that, according to the authors, mutually worsen each other: Alzheimer's disease AND diabetes; cell oxidant stress AND onset of T2D; chronic heart failure AND diabetes; COPD AND T2D; eating disorders AND diabetes; endothelial dysfunction AND T2D; glucotoxicity/ lipotoxicity AND T2D; non-alcoholic fatty liver AND insulin resistance; magnesium deficiency AND T2D; physical inactivity AND gestational diabetes; PI3K/AKT pathway in obesity AND T2D; sle- ep disturbances AND metabolic function.

The numbers of vicious circles are probably so high because diabetes is one of the most commonly researched diseases. On the other hand, T2D can be considered an energetical imbalance of IR and MetS, when one problem brings another. The inhibited ATP production in the MetS stage would be compensated by increased lipid availability (IMCL). T2D would be the decompensated stage when catabolism goes well beyond anabolism. The regulation can provide sufficient fuel for mitochondrial oxidation at only elevated serum glucose levels, which are themselves toxic. Both hyperlipidemia and hyperglycemia are involved in vicious circles, and therefore, without medical intervention, the disease leads to rapid progression and decompensation [13].

The most significant, incurable disease is aging, which limits lifespan and life expectancy. Genetic errors, acute and chronic illnesses further shorten the life expectancy. By the age of 90, cytoplasmic muscle and bone mass reduce on average by nearly half, which is hardly compatible with life. (Few people survive 90 years, who have cytoplasm mass above the average.) In chronic metabolic disorders, the process of cytoplasm losing is accelerated, which also means a shortening of life expectancy. The European Working Group on Sarcopenia in Older People redefined this syndrome. Among other things, declared that T2D also promotes the develop-

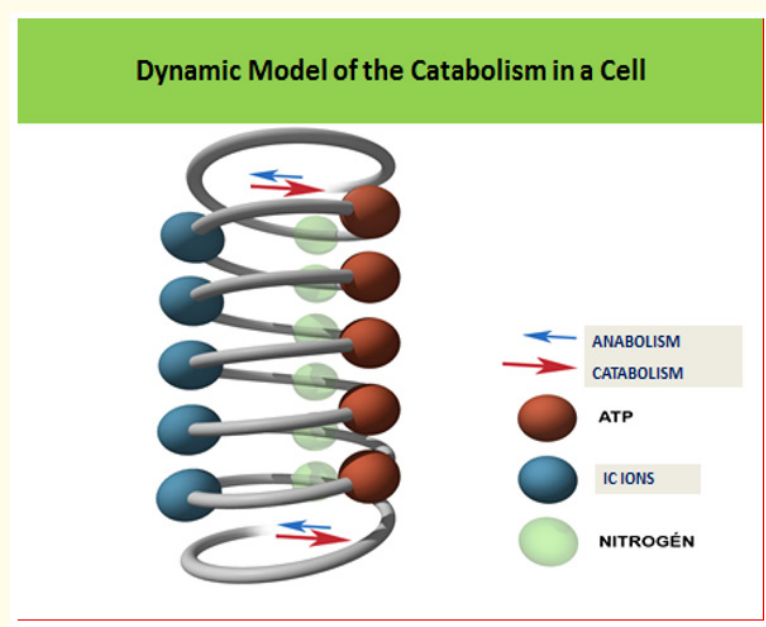

Figure 1: The dynamism of catabolism.

Catabolism is the final merged pathway of the metabolizing cytoplasm: the concentrations of N, ATP and IC ions all decrease, in a parallel fashion.

IC IONS $=$ Cytoplasm Builder Ions. $=$ CBIs

(A vicious circle.) 


\section{Tissues distribution of a healthy human during the life}

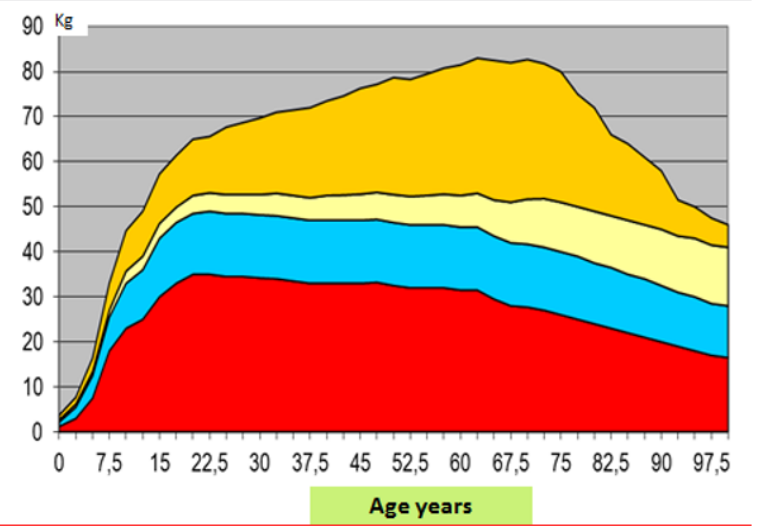

Figure 2: Changes of the three types of tissues and free water and their distribution in a healthy human subject during the life.

\author{
Red=Body Cell Mass (The Functioning Cytoplasm) \\ Blue=Extracellular Water-Spaces Together \\ Yellow=Connective Tissues \\ Ochre=Adipose Tissue.
}

ment of sarcopenia, obesity does not prevent but pushes it. (It is the so-called Sarcopenic Obesity.) Sarcopenia also includes cachexia (which is also possible with obesity). Perhaps it is surprising that T2D goes towards sarcopenia, and it is twice or three times more likely to develop as much as than in simple aging. The poorly managed T2D goes on for years and decades of the muscle and body degradation. (Figure 3). The poorly managed T2D goes on for years and decades of muscle and body degradation.) Even the best-treated T2D patients have faster BCM decrease and shorter life than it would have been without T2D. Counter-regulation varies from person to person, so the extent of the involvement of each organ also varies, that is, the clinical picture will be different.

It is well known that sarcopenia and Sarcopenic Obesity can cause IR [43]. Insulin has an anticatabolic effect, whereas IR and T2D increase protein loss from skeletal muscle and promote the development of sarcopenia [44]. It is also a vicious circle. Muscle mass inversely correlated with mortality in T2D. To increase muscle mass is vital to protect patients against T2D [45]. Although the volume of muscle is deceptive, as the diabetic skeletal muscle has a reduced quality, it contains less cytoplasm and more lipids than a normal one.

It has been suggested that the IMCL is cumulating in the cytoplasm because the lipid cannot burn fast enough $[14,15,46]$. According to others, lipid oxidation is not decreased but is increased, for example, in the myocardium [47]. The truth is probably in the middle: mitochondrial lipid oxidation should compensate the inhibited glucose oxidation; otherwise, T2D would be an acute disorder and not a chronic disease progressively worsening for decades. However, it was shown that, for various, not wholly understood reasons, both glucose and lipid oxidative phosphorylation is impaired. Within specific frameworks, enhanced availability of lipids in IMCL may promote compensatorily, the increased oxidation [46]. Similarly, increased glucose availability (hyperglycemia) in T2D can normalize insulin-stimulated muscle glucose uptake, glycogenesis, and also glucose oxidation [35]. It is a crucial fact that both glucose and lipid availability, even in the case of diabetes, can increase ATP production [36].

To produce "just enough" amount of cytoplasmic ATP is an "alpha command." The cells, the living entity, will undoubtedly generate enough ATP, anyway, if they "want to survive." Glucose oxidation is already inhibited by IR. What is left is the lipids, which can burn and, ultimately, the degradation of cytoplasm. That is why the compensatory role of lipids to provide the necessary amount of ATP is unquestionable during the stage of MetS. When the compensatory function of lipids exhausts, then T2D begins with accelerated catabolism, many vicious circles, which decompensate metabolism toward Sarcopenic Obesity.

Metabolic syndrome and T2D are hypoarousal states and have slightly decelerated metabolism due to slow-grade intracellular acidosis. Besides, even in well-controlled diabetes has no perfectly balanced metabolism, there is a barely visible stealth catabolic overactivity. It follows that Body Cell Mass and Lean Body Mass decrease more during decades than during healthy aging, and therefore life expectancy is reduced [45]. Typically, this applies initially to muscle tissue, but as a complication, almost all organs can be affected; for example, cardiovascular diseases, polyneuropathy, retinopathy, osteopathy frequently develop.

In T2D, there is a damaged hypothalamus insufficient to induce postprandial thermogenesis [17]. The reduced metabolism and energy expenditure are risk factors for body-weight-gain [18] and

Citation: András Sikter. "Psychosomatic Molecular Mechanisms of Metabolic Syndrome and Type 2 Diabetes. Part 3. Is Type 2 Diabetes a Decompensated Form of Metabolic Syndrome?". Acta Scientific Medical Sciences 4.3 (2020): 10-23.

DOI: $10.31080 /$ ASMS.2020.04.0553

Published: February 07, 2020 
T2D. By contrast, in T2D, appetite does not decrease - in line with reduced metabolism - but increases [48]. The physicians often regard this fact to be subjective since the patients have significant energy reserves (fat). However, we can utilize either fat or carbohydrates only after burning them, gaining ATP. As we saw abo-

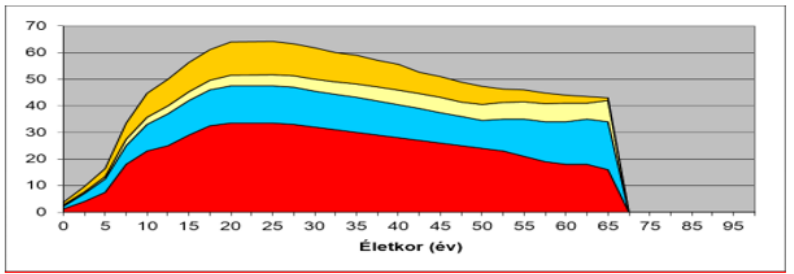

Figure 3A: Malnutrition.

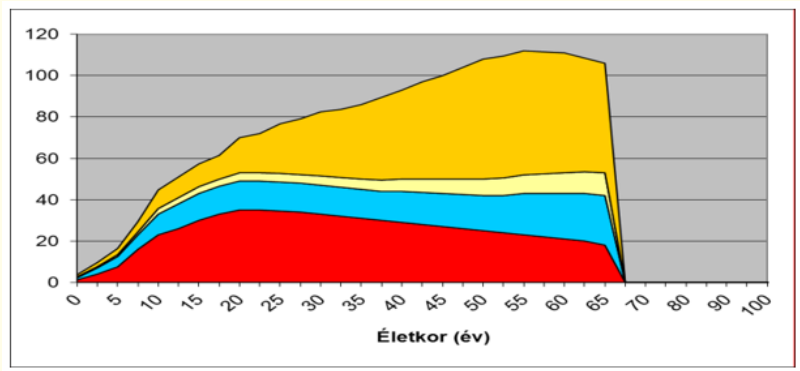

Figure 3B: Poorly treated T2D.

Figure 3: The author has drawn deliberately these figures to be primitive. He wanted to demonstrate that high - grade cytoplasm deficiencies (BCM deficiency = sarcopenia) are similar in both cases. On 3A figure malnourished patient has an identical amount of cytoplasm as the 3B poorly treated T2D patient. A significant difference is only in the fat content (it is 'Sarcopenic Obesity'). It should not be overlooked in the pathophysiology of T2D! According to the two figures, the diseases would have started around the age of 20 .

ve, both glucose and fat oxidation are reduced. The T2D patient is often necessary to uptake more massive amounts of food, to obtain sufficient energy because the efficiency of oxidative phosphorylation is reduced. The calories added, on the other hand, are stored in the form of fat, which further increases IR. (Vicious circle: imperfect oxidative phosphorylation in mitochondria - decreased ATP content in the cells, especially in the hypothalamic hunger center - increased appetite - increased calorie intake - increased lipid tissue of the body - increased insulin resistance - further imperfect mitochondrial working). It is proven that hunger is indeed regulated by the ATP content of hypothalamic cells [49]. ATP-regulated glucose-sensing hypothalamic receptor cells have also been identified in the hunger center [50]. A T2D patient is only able to balance his metabolism often with some self-discipline. Many T2D patients frequently suffer from starvation, and they have experiences that high-calorie intake would reduce their hunger, but they further suffer.

\section{Conclusion}

Insulin, one of the most important hormones, and is one of the most crucial means to regulate metabolism. It controls the level of serum glucose and its uptake into cells having insulin receptors, and it does many more. Through steps not yet known precisely, it increases the cytosolic concentrations of $\mathrm{HPO}_{4}{ }^{2-}$ in cells having insulin receptors and decreases the cytosolic $\mathrm{H}^{+}$concentration by increasing the $\mathrm{pHi}$. The alkaline $\mathrm{pH}$ and elevated $\mathrm{Pi}$ concentration of cytosol trigger the intracellular trafficking of glucose from the membrane to the mitochondria (via GLUT4 activation), while increasing the electrochemical potential of the hydrogen ion of the inner mitochondrial membrane, raising oxidative phosphorylation and ATP formation (see Part 1. and Part 4.). The alkaline $\mathrm{pH}$ of cytosol enhances metabolism, and the equilibrium tilts towards anabolism. The anabolic ("anticatabolic") action of insulin permits cytoplasmic growth and all the physiological processes it facilitates [1]. All of these physiological consequences of insulin can also be interpreted that the effects of the elevated cytosolic $\mathrm{HPO}_{4}{ }^{2-}$ concentration, as a second messenger, are prevailed in the cytoplasm. These data reaffirm the validity of the Momentary Intracellular Ion-Pattern Theory (see Part 1.). At this point, it is not yet clear that insulin will increase the cytosolic concentration of $\mathrm{HPO}_{4}{ }^{2-}$ in one or more steps.

There is consensus that due to IR, which is concluded part of both MetS and T2D, an inhibited mitochondrial oxidative phosphorylation occurs. (The inhibition refers mainly to glucose oxidation but also of lipids). IR may precede T2D for decades [9]. There is no consensus about whether IR is hereditary or acquired, neither in that, it reversible or irreversible changes. And there is no exact scenario, which is the primary cause and which ones are the consequences of the three go together during IR. The triad includes 
increased IMCL, decreased ATP synthesis, and Pi transport was written by Petersen., et al [7]. There is no doubt that irreversible age-related changes impair mitochondrial function, and it is a fact that IR and T2D increase with age. Some [36] doubt that a major, genetically determined cause would be the most significant factor in the pathophysiology of IR. The author of this book thinks neither. T2D is primarily a contemporary pandemic disease, with a low prevalence in earlier centuries. During that time, the genome of humankind had not changed radically. He believes that the most common primary cause of IR is chronic low-grade intracellular acidosis, and the psychosomatic mechanisms play significant roles in its pathophysiology. According to the rules of human coexistence, self-restraint is almost obligatory, the animal model of which is the Social Defeat mechanism (see Part 2 [3]). As a result, low-grade chronic hypercapnia will persist, which can occur in various clinical forms (OSA, OSAS, COPD, Significant Obesity, OHS). Chronic hypercapnia, intracellular acidity, elicits counter-regulation that manifests in the individually-specific MetS-related clinical images, one of them is T2D.

According to the author's theory, one of the most common causes of IR, therefore, is low-grade intracellular acidosis, which impedes the mitochondrial ATP-forming capacity of cells due to the impaired cytosolic phosphate uptake and decreased alkalinization ability of cytoplasm. Briefly, because of the target cells are unable to increase their concentrations of $\mathrm{HPO}_{4}{ }^{2-}$, that is why GLUT4 trafficking and mitochondrial oxidative phosphorylation are hindered. The body (cells) must even provide the increased ATP requirements (see Part 1.), despite the transport and oxidation of glucose are substantially inhibited. The increased availability of lipids compensates hindered glucose oxidation. As a consequence of hyperlipemia and decreased lipid oxidation, the intracellular lipid content increased in several cell types (skeletal muscle cells, liver, and heart). For many years, IMCL can provide enough fuel and compensate for the inhibited glucose oxidation. (Serum lipid levels are a good predictor of IR [7]). When increased lipid availability becomes an inadequate source of energy, and mitochondrial ATP production becomes insufficient, the T2D is starting. Then the body increases the usage up all of the available backup energy: gluconeogenesis increases, the protein breakdown, and amino acids' burning raise, and energy regulation increases their serum levels, food, and calorie uptake also raise, appetite grows. The catabolism of cytoplasm strengthens: the fuel comes from different places to produce the much-needed amount of ATP — the energy imbalance provokes dozens of vicious circles. The above pro and counter regulations affect various organs and cell types differently (see Part 1.: Locus Minoris Resistentiae). Therefore, complications appear as individual patterns. During chronic metabolic disorders, the cytoplasmic mass of the body, the organs, the cells reduce systematically (they are under catabolic process), but not in unison. - it is also the case in T2D.

Following the onset of T2D, several vicious circles manifest (see above), mainly due to T2D being a slow (or fast if it was poorly-controlled) progressive catabolic state. The decompensated process activates vicious circles based on the Second Law of Thermodynamics (see Part 1). A large number of vicious circles known is also attributable to T2D because it is one of the most researched diseases. In other words, vicious circles are also prevalent in other progressive decompensated catabolic conditions (e. g., in heart failure); at most, we are not aware of them. Highlighting some of the vicious circles: Despite the 'overnutrition' (obesity), patients with IR are almost permanently starved, as the lower cytosolic ATP level plays a role in inducing or maintaining hunger [50]. The patient can only temporarily overcome the feeling of hunger with polyphagia. It, in turn, increases obesity and worsens the disease. Sarcopenia causes IR, while poorly treated diabetes results in Sarcopenic Obesity $[44,45]$.

In summary, T2D is a decompensated metabolic disorder due to intracellular acidosis; IR inhibited the oxidative phosphorylation of glucose. Lipids compensate for the lack of fuel for a long time. Then the lipids neither can provide enough matter to produce sufficient ATP to cells' function. That's why catabolism starts breakdown the proteins of the body, the 'last bastion.' It increases the gluconeogenesis, further increases blood glucose levels; insulin secretion is raised, but the amount of energy ingested by food uptake also grows. Medical treatment aims to protect this 'ultimate bastion.' The body's cytoplasm content is degraded slowly (or faster) - depending on the treatment and lifestyle. The lack does not occur homogeneously throughout the body, which results in different cytoplasmic deficiencies in various organs and individuals; that is, the complications will be different. Without a radical turn in the basics of metabolism, we can't expect any improvement.

Citation: András Sikter. "Psychosomatic Molecular Mechanisms of Metabolic Syndrome and Type 2 Diabetes. Part 3. Is Type 2 Diabetes a Decompensated Form of Metabolic Syndrome?". Acta Scientific Medical Sciences 4.3 (2020): 10-23.

DOI: 10.31080 /ASMS.2020.04.0553

Published: February 07, 2020 


\section{Bibliography}

1. Meyts P. "The Insulin Receptor and Its Signal Transduction". Available from Endotext (Internet) (2016).

2. Petersen KF., et al. "Impaired Mitochondrial Activity in the Insulin-Resistant Offspring of Patients with Type 2 Diabetes". The New England Journal of Medicine 350 (2004): 664-671.

3. Petersen KF., et al. "Decreased insulin-stimulated ATP synthesis and phosphate transport in muscle of insulin-resistant offspring of type 2 diabetic parents". PLoS Medicine 2.9 (2005): e233.

4. Bose S., et al. "Metabolic network control of oxidative phosphorylation: multiple roles of inorganic phosphate". The Journal of Biological Chemistry 278.40 (2003): 39155-3965.

5. Cline GW., et al. "Impaired glucose transport as a cause of decreased insulin-stimulated muscle glycogen synthesis in type 2 diabetes". The New England Journal of Medicine 341 (1999): 240-246.

6. Polgreen KE., et al. "Modulation of Pi transport in skeletal muscle by insulin and IGF-1". Biochimica et Biophysica Acta 1223.2 (1994): 279-284.

7. Petersen KF and Shulman GI. "Cellular mechanism of insulin resistance in skeletal muscle". Journal of the Royal Society of Medicine 95.42 (2002): 8-13.

8. Pujia A., et al. "Lipid Oxidation Assessed by Indirect Calorimetry Predicts Metabolic Syndrome and Type 2 Diabetes". Frontiers in Endocrinology 9 (2019): 806.

9. Lillioja S., et al. "Insulin resistance and insulin secretory dysfunction as precursors of non-insulin-dependent diabetes mellitus. Prospective studies of Pima Indians". The New England Journal of Medicine 329.27 (1993): 1988-1992.

10. Goodpaster BH. "Mitochondrial deficiency is associated with insulin resistance”. Diabetes 62.4 (2013): 1032-1035.

11. Ritov VB., et al. "Deficiency of subsarcolemmal mitochondria in obesity and type 2 diabetes". Diabetes 54.1 (2005): 8-14.

12. Lim El., et al. "Measuring the acute effect of insulin infusion on ATP turnover rate in human skeletal muscle using phosphorus-31 magnetic resonance saturation transfer spectroscopy". NMR in Biomedicine 23.8 (2010): 952-957.
13. Bosma M., et al. "Re-evaluating lipotoxic triggers in skeletal muscle: relating intramyocellular lipid metabolism to insulin sensitivity". Progress in Lipid Research 51.1 (2012): 36-49.

14. Poitout V and Robertson RP. "Minireview: Secondary beta-cell failure in type 2 diabetes--a convergence of glucotoxicity and lipotoxicity". Endocrinology 143.2 (2002): 339-342.

15. Koves TR., et al. "Mitochondrial overload and incomplete fatty acid oxidation contribute to skeletal muscle insulin resistance". Cell Metabolism 7.1 (2008): 45-56.

16. Kahn SE. "The relative contributions of insulin resistance and beta-cell dysfunction to the pathophysiology of Type 2 diabetes". Diabetologia 46.1 (2003): 3-19.

17. Schwartz MW., et al. "Central nervous system control of food intake”. Nature 404.6778 (2000): 661-671.

18. Ravussin E., et al. "Reduced rate of energy expenditure as a risk factor for body-weight gain". The New England Journal of Medicine 318.8 (1988): 467-472.

19. Busa WB and Nuccitelli R. "Metabolic regulation via intracellular pH". The American Journal of Physiology 246.4-2 (1984): R409-R438.

20. Gunawardana SC., et al. "Nutrient-stimulated insulin secretion in mouse islets is critically dependent on intracellular $\mathrm{pH}$ ". BMC Endocrine Disorders 4.1 (2004): 1.

21. Juntti-Berggren L., et al. "Glucose-induced increase in cytoplasmic $\mathrm{pH}$ in pancreatic beta-cells is mediated by $\mathrm{Na}+\mathrm{H}+$ exchange, an effect not dependent on protein kinase C". The Journal Biology of Chemistry 266.35 (1991): 23537-23541.

22. Dechant R., et al. "Cytosolic $\mathrm{pH}$ is a second messenger for glucose and regulates the PKA pathway through V-ATPase". The EMBO Journal 29.15 (2010): 2515-2526.

23. Pang H., et al. "Hv1 channel supports insulin secretion in pancreatic $\beta$ cells through calcium entry, depolarization and intracellular pH regulation". (2017).

24. DeCoursey TE. "Voltage and $\mathrm{pH}$ sensing by the voltage-gated proton channel, HV1". Journal of the Royal Society, Interface 15.141 (2018): 20180108.

25. Barg S., et al. "Priming of insulin granules for exocytosis by granular $\mathrm{Cl}(-)$ uptake and acidification". Journal of Cell Science 114.Pt11 (2001): 2145-2154.

Citation: András Sikter. "Psychosomatic Molecular Mechanisms of Metabolic Syndrome and Type 2 Diabetes. Part 3. Is Type 2 Diabetes a Decompensated Form of Metabolic Syndrome?". Acta Scientific Medical Sciences 4.3 (2020): 10-23. 
26. Nabe K., et al. "Diphenylhydantoin suppresses glucose-induced insulin release by decreasing cytoplasmic $\mathrm{H}+$ concentration in pancreatic islets". Endocrinology 147.6 (2006): 2717-2727.

27. Pace CS and Tarvin JT. "pH modulation of glucose-induced electrical activity in B-cells: involvement of $\mathrm{Na} / \mathrm{H}$ and $\mathrm{HCO} 3 /$ $\mathrm{Cl}$ antiporters". The Journal of Membrane Biology 73.1 (1983): 39-49.

28. Baldini N and Avnet S. "The effects of systemic and local acidosis on insulin resistance and signalling". International Journal of Molecular Sciences 20.1 (2018): E126.

29. Igarashi M., et al. "Effect of acidosis on insulin binding and glucose uptake in isolated rat adipocytes". The Tohoku Journal of Experimental Medicine 169.3 (1993): 205-213.

30. Sasaki H., et al. "Insulin resistance in the metabolic acidotic rats: Assessment by glucose clamp technique". Diabetologia 33 (1990): A217.

31. Kraut JA and Madias NE. "Consequences and therapy of the metabolic acidosis of chronic kidney disease". Pediatric $\mathrm{Ne}$ phrology 26.1 (2011): 19-28.

32. Marunaka Y. "The Proposal of Molecular Mechanisms of Weak Organic Acids Intake-Induced Improvement of Insulin Resistance in Diabetes Mellitus via Elevation of Interstitial Fluid pH". International Journal of Molecular Sciences 19.10 (2018): E3244.

33. Adeva MM and Souto G. "Diet-induced metabolic acidosis". Clinical Nutrition 30.4 (2011): 416-421.

34. Trigatti BL and Gerber GE. "The effect of intracellular $\mathrm{pH}$ on long-chain fatty acid uptake in 3T3-L1 adipocytes: evidence that uptake involves the passive diffusion of protonated longchain fatty acids across the plasma membrane". Biochemical Journal 313 (1996): 487-497.

35. Kelley DE and Mandarino LJ. "Hyperglycemia normalizes insulin-stimulated skeletal muscle glucose oxidation and storage in noninsulin-dependent diabetes mellitus". The Journal of Clinical Investigation 86.6 (1990): 1999-2007.

36. Szendroedi J., et al. "Muscle mitochondrial ATP synthesis and glucose transport/phosphorylation in type 2 diabetes". PLoS Medicine 4.5 (2007): e154.
37. Beisel WR. "Herman Award Lecture infection-induced malnutrition--from cholera to cytokines". The American Journal of Clinical Nutrition 62.4 (1995): 813-819.

38. Anwar A., et al. "Tumor Necrosis Factor- $\alpha$ Regulates InsulinLike Growth Factor-1 and Insulin-Like Growth Factor Binding Protein-3 Expression in Vascular Smooth Muscle". Circulation 105 (2002): 1220-1225.

39. Korponay TC., et al. "High CO2 downregulates skeletal muscle protein anabolism via AMPK $\alpha 2$-mediated depressed ribosomal biogenesis". American Journal of Respiratory Cell and Molecular Biology (2019).

40. Wachman A and Bernstein DS. "Diet and osteoporosis". Lancet 1.7549 (1968): 958-959.

41. Relman AS., et al. "Endogenous production of fixed acid and the measurement of the net balance of acid in normal subjects". Journal of Clinical Investigation 40 (1961): 1621-1630.

42. Dechant R., et al. "Cytosolic pH regulates cell growth through distinct GTPases, Arf1 and Gtr1, to promote Ras/PKA and TORC1 activity". Molecular Cell 55.3 (2014): 409-421.

43. Srikanthan P., et al. "Sarcopenia exacerbates obesity-associated insulin resistance and dysglycemia: findings from the National Health and Nutrition Examination Survey III". PLoS One 5.5 (2010): e10805.

44. Guillet $\mathrm{C}$ and Boirie Y. "Insulin resistance: a contributing factor to age-related muscle mass loss?" Diabetes and Metabolism 31.2 (2005): 5S20-5S26.

45. Miyake H., et al. "Low skeletal muscle mass is associated with the risk of all-cause mortality in patients with type 2 diabetes mellitus". Therapeutic Advances in Endocrinology and Metabolism 10 (2019).

46. Aon MA., et al. "Mitochondrial and cellular mechanisms for managing lipid excess". Frontiers in Physiology 5 (2014): 282.

47. Abel ED. "Free fatty acid oxidation in insulin resistance and obesity". Heart and Metabolism: Management of the Coronary Patient 48 (2010): 5-10.

48. Slyper AH. "A paradigm shift for the prevention and treatment of individual and global obesity". Diabetes Metabolic Syndrome and Obesity: Targets and Therapy 11 (2018): 855-861.

Citation: András Sikter. "Psychosomatic Molecular Mechanisms of Metabolic Syndrome and Type 2 Diabetes. Part 3. Is Type 2 Diabetes a Decompensated Form of Metabolic Syndrome?". Acta Scientific Medical Sciences 4.3 (2020): 10-23.

DOI: $10.31080 /$ ASMS.2020.04.0553

Published: February 07, 2020 
Psychosomatic Molecular Mechanisms of Metabolic Syndrome and Type 2 Diabetes. Part 3. Is Type 2 Diabetes a Decompensated Form of Metabolic Syndrome?

49. MacLean DB and Luo LG. "Increased ATP content/production in the hypothalamus may be a signal for energy-sensing of satiety: studies of the anorectic mechanism of a plant steroidal glycoside". Brain Research 1020.1-2 (2004): 1-11.

50. Bolborea M and Dale N. "Hypothalamic tanycytes: potential roles in the control of feeding and energy balance". Trends in Neurosciences 36.2 (2015): 91-100.

\section{Assets from publication with us}

- Prompt Acknowledgement after receiving the article

- Thorough Double blinded peer review

- Rapid Publication

- Issue of Publication Certificate

- High visibility of your Published work

Website: https://www.actascientific.com/

Submit Article: https://www.actascientific.com/submission.php

Email us: editor@actascientific.com

Contact us: +919182824667

Citation: András Sikter. "Psychosomatic Molecular Mechanisms of Metabolic Syndrome and Type 2 Diabetes. Part 3. Is Type 2 Diabetes a Decompensated Form of Metabolic Syndrome?". Acta Scientific Medical Sciences 4.3 (2020): 10-23.

DOI: $10.31080 /$ ASMS.2020.04.0553

Published: February 07, 2020 